\title{
End-to-End Trainable Non-Collaborative Dialog System
}

\author{
Yu Li, Kun Qian, Weiyan Shi, Zhou Yu \\ University of California, Davis \\ \{yooli, kunqian, wyshi, joyu\}@ucdavis.edu
}

\begin{abstract}
End-to-end task-oriented dialog models have achieved promising performance on collaborative tasks where users willingly coordinate with the system to complete a given task. While in non-collaborative settings, for example, negotiation and persuasion, users and systems do not share a common goal. As a result, compared to collaborate tasks, people use social content to build rapport and trust in these non-collaborative settings in order to advance their goals. To handle social content, we introduce a hierarchical intent annotation scheme, which can be generalized to different non-collaborative dialog tasks. Building upon TransferTransfo (Wolf et al. 2019), we propose an end-to-end neural network model to generate diverse coherent responses. Our model utilizes intent and semantic slots as the intermediate sentence representation to guide the generation process. In addition, we design a filter to select appropriate responses based on whether these intermediate representations fit the designed task and conversation constraints. Our noncollaborative dialog model guides users to complete the task while simultaneously keeps them engaged. We test our approach on our newly proposed ANTISCAM dataset and an existing PersuasionForGood dataset. Both automatic and human evaluations suggest that our model outperforms multiple baselines in these two non-collaborative tasks.
\end{abstract}

\section{Introduction}

Considerable progress has been made building end-to-end dialog systems for collaborative tasks in which users cooperate with the system to achieve a common goal. Examples of collaborative tasks include making restaurant reservations and retrieving bus time-table information. Since users typically have clear and explicit intentions in collaborative tasks, existing systems commonly classify user utterances into predefined intents. In contrast, non-collaborative tasks are those where the users and the system do not strive to achieve the same goal. Examples of such tasks include deceiving attackers, persuading users to donate to a cause (Wang et al. 2019), and negotiating a product price (He et al. 2018; Cao et al. 2018). In these tasks, users often perform complex actions that are beyond a simple set of pre-defined in-

Copyright (C) 2020, Association for the Advancement of Artificial Intelligence (www.aaai.org). All rights reserved. tents. In order to reach a common state, the user and the system need to build rapport and trust which naturally involves off-task content. Previous work did not model offtask content (He et al. 2018), which may have led to less optimal results. For example, in the persuasion task (Wang et al. 2019), users would ask the system "How do you feel about war?" An example of an on-task system response that the system could have made is "Do you want to make a donation?", which sticks to the task but neglects users' question. However, a better response to such an off-task question is "War is destructive and pitiless, but you can donate to help child victims of war." This response is better, as it has been found that users are more likely to end the conversation if the system neglects their questions (Yu, Black, and Rudnicky 2017). Therefore, we need to design a system that handles both on-task and off-task information appropriately and in a way that leads back to the system's goal.

To tackle the issue of incoherent system responses to offtask content, previous studies have built hybrid systems to interleave off-task and on-task content. Yu, Black, and Rudnicky used a rule-based dialog manager for on-task content and a neural model for off-task content, and trained a reinforcement learning model to select between these two models based on the dialog context. However, such a method is difficult to train and struggles to generalize beyond the movie promotion task they considered. To tackle these problems, we propose a hierarchical intent annotation scheme that separates on-task and off-task information in order to provide detailed supervision. For on-task information, we directly use task-related intents for representation. Off-task information, on the other hand, is too general to categorize into specific intents, so we choose dialog acts that convey syntax information. These acts, such as "open question" are general to all tasks.

Previous studies use template-based methods to maintain sentence coherence. However, rigid templates lead to limited diversity, causing the user losing engagement. On the other hand, language generation models can generate diverse responses but are bad at being coherent. We propose Multiple Intents and Semantic Slots Annotation Neural Network (MISSA) to combine the advantages of both template and generation models and takes advantage from the hier- 
archical annotation at the same time. MISSA follows the TransferTransfo framework (Wolf et al. 2019) with three modifications: (i) We first concurrently predict user's, system's intents and semantic slots; (ii) We then perform conditional generation to improve generated response's coherence. Specifically, we generate responses conditioned on the above intermediate representation (intents and slots); (iii) Finally, we generate multiple responses with the nucleus sampling strategy (Holtzman et al. 2019) and then apply a response filter, which contains a set of pre-defined constraints to select coherent responses. The constraints in the filter can be defined according to specific task requirements or general conversational rules.

To enrich publicly available non-collaborative task datasets, we collect a new dataset ANTISCAM, where users defend themselves against attackers trying to collect personal information. As non-collaborative tasks are still relatively new to the study of dialog systems, there are insufficiently many meaningful datasets for evaluation and we hope this provides a valuable example. We evaluate MISSA on the newly collected ANTISCAM dataset and an existing PersuasionForGood dataset. Both automatic and human evaluations suggest that MISSA outperforms multiple competitive baselines.

In summary, our contributions include: (i) We design a hierarchical intent annotation scheme and a semantic slot annotation scheme to annotate the non-collaborative dialog dataset, we also propose a carefully-designed ANTISCAM dataset to facilitate the research of non-collaborative dialog systems. (ii) We propose a model that can be applied to all non-collaborative tasks, outperforming other baselines on two different non-collaborative tasks. (iii) We develop an anti-scam dialog system to occupy attacker's attention and elicit their private information for social good. Furthermore, we also build a persuasion dialog system to persuade people to donate to charities. We release the code and data. ${ }^{1}$

\section{Related Work}

The interest in non-collaborative tasks has been increasing and there have already been several related datasets. For instance, Wang et al. (2019) collected conversations where one participant persuades another to donate to a charity. He et al. (2018) collected negotiation dialogs where buyers and sellers bargain for items for sale on Craigslist. There are many other non-collaborative tasks, such as the turntaking game (DeVault, Mell, and Gratch 2015), the multiparty game (Asher et al. 2016) and item splitting negotiation (Potts 2012). Similar to the ANTISCAM dataset proposed in this paper, these datasets contain off-task content and can be used to train non-collaborative dialog systems. However, since they are not specifically collected and designed for non-collaborative tasks, it might be difficult to disentangle the on-task and off-task contents and measure the performance. Therefore, we propose the ANTISCAM dataset, which is designed to interleave the on-task and offtask contents in the conversation, and can serve as a benchmark dataset for similar non-collaborative tasks.

\footnotetext{
${ }^{1}$ https://gitlab.com/ucdavisnlp/antiscam
}

To better understand user utterances and separate ontask and off-task content within a conversation, previous work has designed hierarchical annotation schemes for specific domains. Hardy et al. (2002) followed the DAMSL scheme(Allen and Core 1997) and annotated a multilingual human-computer dialog corpus with a hierarchical dialog act annotation scheme. Gupta et al. (2018) used a hierarchical annotation scheme for semantic parsing. Inspired by these studies, our idea is to annotate the intent and semantic slot separately in non-collaborative tasks. We propose a hierarchical intent annotation scheme that can be adopted by all non-collaborative tasks. With this annotation scheme, MISSA is able to quickly build an end-to-end trainable dialog system for any non-collaborative task.

Traditional task-oriented dialog systems (Young et al. 2013) are usually composed of multiple independent modules, for example, natural language understanding, dialog state tracking (Williams, Raux, and Henderson 2016; Mrkšić et al. 2016), dialog policy manager (Levin, Pieraccini, and Eckert 2000), and natural language generation (Lei et al. 2018). Conversational intent is adopted to capture the meaning of task content in these dialog systems (He et al. 2018; Zhao, Zhao, and Eskenazi 2017). In comparison to this work, we use a hierarchical intent scheme that includes off-task and on-task intents to capture utterance meaning. We also train the model in a multi-task fashion to predict decoupled intents and semantic slots. The major defect of a separately trained pipeline is the laborious dialog state design and annotation. In order to mitigate this problem, recent work has explored replacing independent modules with end-to-end neural networks (Wen et al. 2016; Williams, Asadi, and Zweig 2017; Liang et al. 2019). Our model also follows this end-to-end fashion.

Over the last few years, we have witnessed a huge growth in non-task-oriented dialog systems (Vinyals and Le 2015; Li et al. 2016). Social chatbots such as Gunrock (Chen et al. 2018) were able to maintain a conversation for around ten minutes in an open domain. Recent improvements build on top of the transformer and pre-trained language models (Devlin et al. 2018; Yang et al. 2019; Radford et al. 2019), obtained state-of-the-art results on the PERSONACHAT dataset (Wolf et al. 2019). Pre-trained language models are proposed to build task-oriented dialog systems to drive the progress on leveraging large amounts of available unannotated data. (Budzianowski and Vulić 2019). Similarly, our approach is also built on top of the TransferTransfo framework (Wolf et al. 2019). Budzianowski and Vulić (2019) focused on collaborative tasks (Budzianowski et al. 2018). We target non-collaborative tasks instead.

Another line of work interleaves on-task and off-task content by building a hybrid dialog system that combines a task-oriented model and a non-task-oriented model (Yu, Black, and Rudnicky 2017; Papaioannou et al. 2017). In these studies, task-oriented systems and non-task-oriented systems are designed separately and both systems generate candidate responses. A selector is then designed to choose an appropriate output from the candidate responses ( $\mathrm{Yu}$, Black, and Rudnicky 2017) and a connector to combine two response candidates (Zhao, Romero, and Rudnicky 2018; 
Baheti et al. 2018). Compared with these works, MISSA is end-to-end trainable and thus easier to train and update.

\begin{tabular}{|c|c|c|}
\hline \multirow{12}{*}{ On-task } & \multirow{3}{*}{ ANTISCAM } & elicitation \\
\hline & & providing_information \\
\hline & & refusal \\
\hline & \multirow{9}{*}{$\begin{array}{l}\text { PERSUASION- } \\
\text {-FORGOOD }\end{array}$} & agree_donation \\
\hline & & disagree_donation \\
\hline & & disagree_donation_more \\
\hline & & ask_donation_amount \\
\hline & & ask_donate_more \\
\hline & & proposition_of_donation \\
\hline & & er_confirm_donation \\
\hline & & ee_confirm_donation \\
\hline & & provide_donation_amount \\
\hline \multirow{12}{*}{\multicolumn{2}{|c|}{ Off-task }} & open_question \\
\hline & & yes_no_question \\
\hline & & negative_answer \\
\hline & & positive_answer \\
\hline & & responsive_statement \\
\hline & & nonresponsive_statement \\
\hline & & greeting \\
\hline & & thanking \\
\hline & & respond_to_thank \\
\hline & & apology \\
\hline & & closing \\
\hline & & hold \\
\hline
\end{tabular}

Table 1: Hierarchical intent annotation scheme on both ANTISCAM dataset and PERSUASIONFOrGOOD dataset. The On-task intents are task-specific while the Off-task intents are general for different non-collaborative tasks.

\section{Non-Collaborative Task Annotation Scheme}

To decouple syntactic and semantic information in utterances and provide detailed supervision, we design a hierarchical intent annotation scheme for non-collaborative tasks. We first separate on-task and off-task intents. As on-task intents are key actions that can vary among different tasks, we need to specifically define on-task intents for each task. On the other hand, since off-task content is too general to design task-specific intents, we choose common dialog acts as the categories. The advantage of this hierarchical annotation scheme is apparent when starting a new non-collaborative task: we only need to focus on designing the on-task categories and semantic slots which are the same as traditional task-oriented dialog systems. Consequently, we don't have to worry about the off-task annotation design since the offtask category is universal.

In the intent annotation scheme shown in Table 1, we list the designed intent annotation scheme for the newly collected ANTISCAM dataset and the PERSUASIONFORGood dataset. We first define on-task intents for the datasets, which are key actions in the task. Since our ANTISCAM focuses on understanding and reacting towards elicitations, we define elicitation, providing_information and refusal as on-task intents. In the PERSUASIONFOR-
GooD dataset, we define nine on-task intents in Table 1 based on the original PERSUASIONFORGOOD dialog act annotation scheme. All these intents are related to donation actions, which are salient on-task intents in the persuasion task. The off-task intents are the same for both tasks, including six general intents and six additional social intents. General intents are more closely related to the syntactic meaning of the sentence (open_question, yes_no_question, positive_answer, negative_answer, responsive_statement, and nonresponsive_statement) while social intents are common social actions (greeting, closing, apology, thanking,respond_to_thank, and hold).

For specific tasks, we also design a semantic slot annotation scheme for annotating sentences based on their semantic content. We identify 13 main semantic slots in the anti-scam task, for example, credit card numbers. We present a detailed semantic slot annotation in Table 2. Following Wang et al., we segment each conversation turn into single sentences and then annotate each sentence rather than turns.

\begin{tabular}{ll}
\hline Annotation & Examples \\
\hline \hline order_detail & "Your order will arrive by Thursday afternoon." \\
order_update & "Should I cancel your order?" \\
payment & "Was payment made with a Visa card?" \\
name & "Can you give me your name?" \\
identity & "I am from Amazon customer service." \\
address & "Can confirm your billing address for me?" \\
phone_num & "What is your phone number?" \\
card_info & "I need the credit card info please" \\
card_num & "Can you verify your card number for me?" \\
card_cvs & "Next, I will need the CVS number from that card." \\
card_date & "Can I have expiration date?" \\
account_detail & "This is to confirm your account." \\
others & "How are you doing?" \\
\hline
\end{tabular}

Table 2: ANTISCAM's semantic slot annotation scheme.

\section{Datasets}

We test our approach on two non-collaborative task datasets: the ANTISCAM dataset and the PERSUASIONFORGOOD dataset (Wang et al. 2019). Both datasets are collected from the Amazon Mechanical Turk platform in the form of typing conversations and off-task dialog is interleaved in the dialog.

\section{AnTISCAM Dataset}

To enrich available non-collaborative task datasets, we created a corpus of human-human anti-scam dialogs in order to learn human elicitation strategies. We chose a popular Amazon customer service scam scenario to collect dialogs between users and attackers who aim to collect users information. We posted a role-playing task on the Amazon Mechanical Turk platform and collected a typing conversation dataset named ANTISCAM. We collected 220 human-human dialogs. The average conversation length is 12.45 turns and the average utterance length is 11.13 words. Only 172 out of 220 users successfully identified their partner as an attacker, suggesting that the attackers are well trained and not too easily identifiable. We recruited two expert annotators who 
have linguistic training to annotate 3,044 sentences in 100 dialogs, achieving a 0.874 averaged weighted kappa value.

\section{PersuasionForGood Dataset}

The PersuasionForGood dataset (Wang et al. 2019) was collected from typing conversations on Amazon Mechanical Turk platform. Two workers were randomly paired, one was assigned the role of persuader, the other was persuadee. The goal of the persuader was to persuade the persuadee to donate a portion of task earning to a specific charity. The dataset consists of 1,017 dialogs, where 300 dialogs are annotated with dialog acts. The average conversation length is 10.43 , the vocabulary size is 8,141 . Since the original PERSUASIONFORGOOD dataset is annotated with dialog acts, we select the on-task dialog acts as on-task intents shown in Table 1, and categorize the other dialog acts into our predefined off-task intents.

\section{Model}

\section{Background}

The TransferTransfo framework was proposed to build open domain dialog systems. Wolf et al. (2019) fine-tuned the generative pre-training model (GPT) (Radford et al. 2018) with the PERSONA-CHAT dataset (Zhang et al. 2018) in a multi-task fashion, where the language model objective is combined with a next-utterance classification task. The language model's objective is to maximize the following likelihood for a given sequence of tokens, $X=\left\{x_{1}, \ldots, x_{n}\right\}$ :

$$
\mathcal{L}_{L M}(X)=\sum_{i=1}^{n} \log P\left(x_{i} \mid x_{0}, \ldots, x_{i-1}\right)
$$

The authors also trained a classifier to distinguish the correct next-utterance appended to the input human utterances from a set of randomly selected utterance distractors. In addition, they introduced dialog state embeddings to indicate speaker role in the model. The model significantly outperformed previous baselines over both automatic evaluations and human evaluations in social conversations. Since the TransferTransfo framework performs well in open domain, we adapt it for non-collaborative settings. We keep all the embeddings in the framework and train the language model and next-utterance classification task in a multi-task fashion following TransferTransfo.

We make two major changes: (1) To address the problem that TransferTransfo is originally designed for an open domain without explicit intents and regulations, we add two intent classifiers and two semantic slot classifiers to classify the intents and semantic slots for both human utterances and system responses as an effort to incorporate the proposed hierarchical intent and semantic slot annotation for non-collaborative tasks. (2) In dialog systems, multiple generated responses can be coherent under the current context. Generating diverse responses has proven to be an enduring challenge. To increase response diversity, we sample multiple generated responses and choose an appropriate one according to a set of pre-defined rules.

\section{Intent and Semantic Slot Classifiers}

We train MISSA in a multi-task fashion. In addition to the language model task and the next-utterance prediction task, we also use separate classifiers to predict the intents and semantic slots of both human utterances and system responses. The intent classifier and semantic slot classifier for human utterances capture the semantic and syntactic meaning of human utterances, providing information to select the appropriate response among response candidates while the classifiers for the system intents and semantic slots are designed to help select an appropriate next-sentence. We describe response filtering in the corresponding subsection. Classifiers are designed as the following equation:

$$
p\left(L_{t}^{i}\right)=\operatorname{softmax}\left(\left[\begin{array}{l}
h_{t-1}^{l} \\
h_{t}^{i}
\end{array}\right] \cdot W_{2 h}\right)
$$

where $L_{t}^{i}$ is the intent or semantic label of $i$-th sentence at turn $t . h_{t-1}^{l}$ is the hidden states at the end of last sentence in turn $t-1, h_{t}^{i}$ is the last hidden states at the end of $i$-th sentence in turn $t$. $W_{2 h}$ are weights learned during training.

MISSA is able to classify multiple intents and multiple semantic slots in a single utterance with these classifiers. Figure 1 shows how it works on the ANTISCAM dataset. Specifically, we set a special token $\langle$ sep $>$ at the end of each sentence in an utterance (an utterance can consist of multiple sentences). Next, we pass the token's position information to the transformer architecture and obtain the representation of the position (represented as colored position at last layer in Figure 1). After that, we concatenate the embeddings at these position with the hidden states of last sentence. We pass these concatenated representations to the intent classifier and the slot classifier to obtain an intent and a semantic slot for each sentence in the utterance. As shown in Figure 1 , the loss function $\mathcal{L}$ for the model combines all the task losses:

$$
\begin{aligned}
\mathcal{L}= & \lambda_{L M} \mathcal{L}_{L M}+\lambda_{I_{h}} \mathcal{L}_{I_{h}}+\lambda_{S_{h}} \mathcal{L}_{S_{h}}+\lambda_{I_{s}} \mathcal{L}_{I_{s}} \\
& +\lambda_{S_{s}} \mathcal{L}_{S_{s}}+\lambda_{\text {nup }} \mathcal{L}_{\text {nup }}
\end{aligned}
$$

where $\mathcal{L}_{L M}$ is the language model loss, $\mathcal{L}_{I_{h}}, \mathcal{L}_{S_{h}}, \mathcal{L}_{I_{s}}$, and $\mathcal{L}_{S_{s}}$ are losses of intent and slots classifiers, $\mathcal{L}_{\text {nup }}$ is nextutterance classification loss. $\lambda_{L M}, \lambda_{I_{h}}, \lambda_{S_{h}}, \lambda_{I_{s}}, \lambda_{S_{s}}$, and $\lambda_{\text {nup }}$ are the hyper-parameters that control the relative importance of every loss.

\section{Response Generation}

MISSA can generate multiple sentences in a single system turn. Therefore, we perform system generation conditioned on predicted system intents. More specifically, during the training phase, in addition to inserting a special $<$ sep $>$ token at the end of each sentence, we also insert the intent of the system response as special tokens at the head of each sentence in the system response. For example, in Figure 1, we insert a $<$ pos_ans $>$ token at the head of $S_{t}^{1}$, which is the system response in green. We then use a cross entropy loss function to calculate the loss between the predicted token and the ground truth intent token. During the testing phase, the model first generates a special intent token, then after 


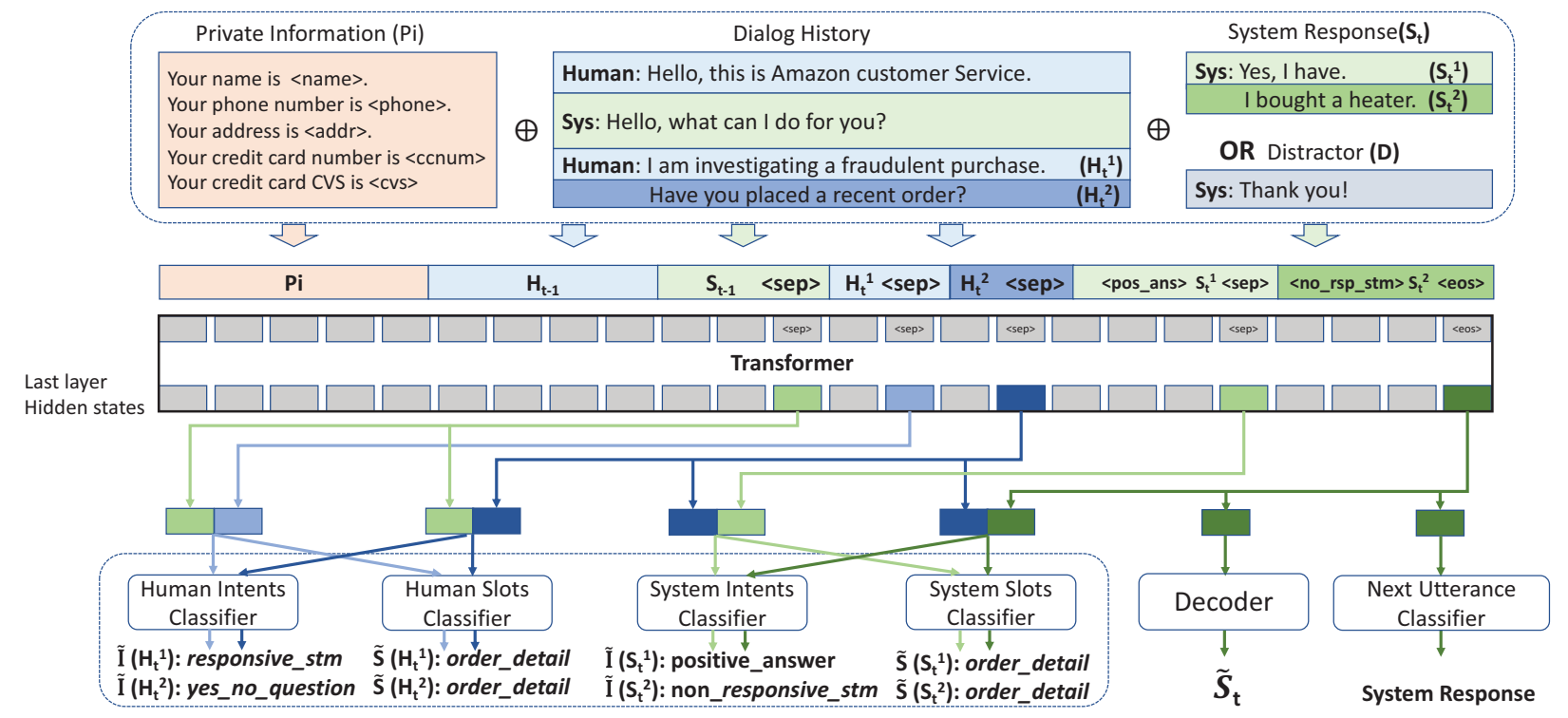

Figure 1: The training phase overview of MISSA on ANTISCAM dataset, the input consists of three parts: private information, dialog history, and an appended next utterance. We concatenate the last hidden states at $<\operatorname{sep}>$ tokens with the last hidden states at the end of the last utterance to predict intents and semantic slots for corresponding sentences. We can predict multiple intents and semantic slots for each human utterance and system response. During testing, the appended response and distractor are removed.

being conditioned on this intent token, the model keeps generating a sentence until it generates a $<s e p>$ token. After that, the model continues to generate another intent token and another sentence until it generates an $\langle e o s>$ token.

\section{Response Filtering}

Since we only perform conditional generation, a type of soft constraint on the predicted intent of system response, the system can still generate samples that violate simple conversation regulations, such as eliciting information that has already been provided. These corner cases may lead to fatal results in high-risk tasks, for example, health care and education. To improve the robustness of MISSA and improve its ability to generalize to more tasks, we add a response filtering module after the generation. With the nucleus sampling strategy (Holtzman et al. 2019), MISSA is able to generate multiple diverse candidate responses with different intents and semantic slots. We then adopt a task-specific response filtering policy to choose the best candidate response as the final output. In our anti-scam scenario, we set up a few simple rules to filter out some unreasonable candidates, for instance, eliciting the repeated information. The filtering module is easily adaptable to different domains or specific requirements, which makes our dialog system more controllable.

\section{Experiments}

We evaluate MISSA on two non-collaborative task datasets. ANTISCAM aims to build a dialog system that occupies the attacker's attention and elicits the attacker's information while PersuasionForGood (Wang et al. 2019) aims to build a dialog system that persuades people to donate to a charity. We use $80 \%$ data for training, $10 \%$ data for validation, and $10 \%$ data for testing. More training details are presented in Appendix.

\section{Baseline Models}

We compare MISSA mainly with two baseline models:

- TransferTransfo The vanilla TransferTransfo framework is compared with MISSA to show the impact and necessity of adding the intent and slot classifiers. We follow the original TransferTransfo design (Wolf et al. 2019) and train with undelexicalized data.

- Hybrid Following Yu, Black, and Rudnicky (2017), we also build a hybrid dialog system by combining vanilla TransferTransfo and MISSA. Specifically, we first determine if the human utterances are on-task or off-task with human intent classifier. If the classifier decides that the utterance is on-task, we choose the response from MISSA; otherwise, we choose the response from vanilla TransferTransfo baseline.

In addition, we perform ablation studies on MISSA to show the effects of different components.

- MISSA-sel denotes MISSA without response filtering.

- MISSA-con denotes MISSA leaving out the intent token at the start of the response generation.

\section{Automatic Evaluation Metrics}

Perplexity Since the canonical measure of a good language model is perplexity, which indicates the error rate of the ex- 


\begin{tabular}{l|ccccc|ccccc}
\hline \multirow{2}{*}{ Model } & \multicolumn{4}{|c|}{ Automatic Evaluation Metrics } & \multicolumn{4}{c}{ Human Evaluation Metrics } \\
& PPL & RIP & RSP & ERIP & ERSP & Fluency & Coherence & Engagement & Length & TaskSuc \\
\hline \hline TransferTransfo & 32.96 & $34.8 \%$ & $46.0 \%$ & $\mathbf{4 8 . 0 \%}$ & $56.3 \%$ & 3.48 & 2.85 & 2.68 & 8.5 & 1.025 \\
\hline Hybrid & - & $32.0 \%$ & $44.0 \%$ & $45.7 \%$ & $55.3 \%$ & 3.25 & 2.76 & 2.60 & 8.2 & 0.975 \\
\hline MISSA & $\mathbf{2 1 . 0 7}$ & $\mathbf{3 5 . 1 \%}$ & $\mathbf{4 6 . 6 \%}$ & $47.2 \%$ & $\mathbf{5 8 . 6 \%}$ & $\mathbf{4 . 1 8}$ & $\mathbf{3 . 7 5}$ & 3.69 & $\mathbf{1 4 . 9}$ & 1.294 \\
\hline MISSA-sel & 30.54 & $31.6 \%$ & $42.4 \%$ & $44.2 \%$ & $53.8 \%$ & 3.60 & 2.92 & 2.87 & 9.9 & 1.000 \\
\hline MISSA-con & 24.46 & $33.8 \%$ & $45.6 \%$ & $46.0 \%$ & $57.3 \%$ & 3.78 & 3.68 & $\mathbf{3 . 7 8}$ & 14.8 & $\mathbf{1 . 3 4 1}$ \\
\hline
\end{tabular}

Table 3: Experiments results with both automatic and human evaluation on ANTISCAM dataset.

pected word. We choose perplexity to evaluate the model performance.

Response-Intent Prediction (RIP) \& Response-Slot Prediction (RSP) Different from open-domain dialog systems, we care about the intents of the system response in noncollaborative tasks as we hope to know if the system response satisfies user intents. For example, in the anti-scam task, if the attacker elicits information from the system, we need to know if the system refuses or agrees to provide the information. Therefore we care about intent prediction for the generated system response. Since our baselines are more suited for social chat as they cannot produce system intents, we use the system intent and slot classifiers trained in our model to predict their responses' intents and slots. The intent predictor achieves a $84 \%$ accuracy and the semantic slot predictor achieves $77 \%$ on the ANTISCAM dataset. Then we compare the predicted values with human-annotated ground truth in the dataset to compute the response-intent prediction (RIP) and response-slot prediction (RSP).

Extended Response-Intent Prediction (ERIP) \& Extended Response-Slot Prediction (ERSP) With ResponseIntent Prediction, we verify the predicted intents to evaluate the coherence of the dialog. However, the real mapping between human-intent and system-intent is much more complicated as there might be multiple acceptable system-intents for the same human-intent. Therefore, we also design a metric to evaluate if the predicted system-intent is in the set of acceptable intents. Specifically, we estimate the transition probability $p\left(I_{i} \mid I_{j}\right)$ by counting the frequency of all the bigram human-intent and system-intent pairs in the training data. During the test stage, if the predicted intent matches the ground truth, we set the score as 1, otherwise we set the score as $p\left(I_{\text {predict }} \mid I_{i}\right)$ where $I_{i}$ is the intent of the input human utterance. We then report the average value of those scores over turns as the final extended response-intent prediction result.

\section{Human Evaluation Metrics}

Automatic metrics only validate the system's performance on a single dimension at a time. The ultimate holistic evaluation should be conducted by having the trained system interact with human users. Therefore we also conduct human evaluations for the dialog system built on ANTISCAM. We test our models and baselines with 15 college-student volunteers. Each of them is asked to pretend to be an attacker and interact with all the models for at least three times to avoid randomness. We in total collect 225 number of dialogs. Each time, volunteers are required to use similar sentences and strategies to interact with all five models and score each model based on the metrics listed below at the end of the current round. Each model receives a total of 45 human ratings, and the average score is reported as the final humanevaluation score. In total, we design five different metrics to assess the models' conversational ability whilst interacting with humans. The results are shown in Table 3.

Fluency Fluency is used to explore different models' language generation quality.

Coherence Different from single sentence's fluency, coherence focuses more on the logical consistency between sentences in each turn.

Engagement In the anti-scam scenario, one of our missions is to keep engaging with the attackers to waste their time. So we directly ask volunteers (attackers) to what extend they would like to continue chatting with the system.

Dialog length (Length) Engagement is a subjective metric. Anti-scam system's goal is to engage user in the conversation longer in order to limit their harm to other potential victims. So we count the dialog length as another metric to evaluate system performance.

Task Success Score (TaskSuc) The other goal of the antiscam system is to elicit attacker's personal information. We count the average type of information (name, address and phone number) that the system obtained from attackers as the task success score.

\section{Results and Analysis}

Table 3 presents the main experiment results on ANTISCAM dataset, for both automatic evaluation metrics and human evaluation metrics. The experiment results on PERSUASIONFORGOOD are shown in Table 5. We observe that MISSA outperforms two baseline models (TransferTransfo and hybrid model) on almost all the metrics on both datasets. For further analysis, examples of real dialogs from the human evaluation are presented in Table 4.

Compared to the first TransferTransfo baseline, MISSA outperforms the TransferTransfo baseline on the on-task contents. From Table 3, we observe that MISSA maintains longer conversations (14.9 turns) compared with TransferTransfo ( 8.5 turns), which means MISSA is better at maintaining the attacker's engagement. MISSA also has a higher task success score (1.294) than TransferTransfo (1.025), which indicates that it elicits information more strategically. In the top two dialogs (A and B) that are shown in Table 4, both attackers were eliciting a credit card number in their first turns. TransferTransfo directly gave away the informa- 


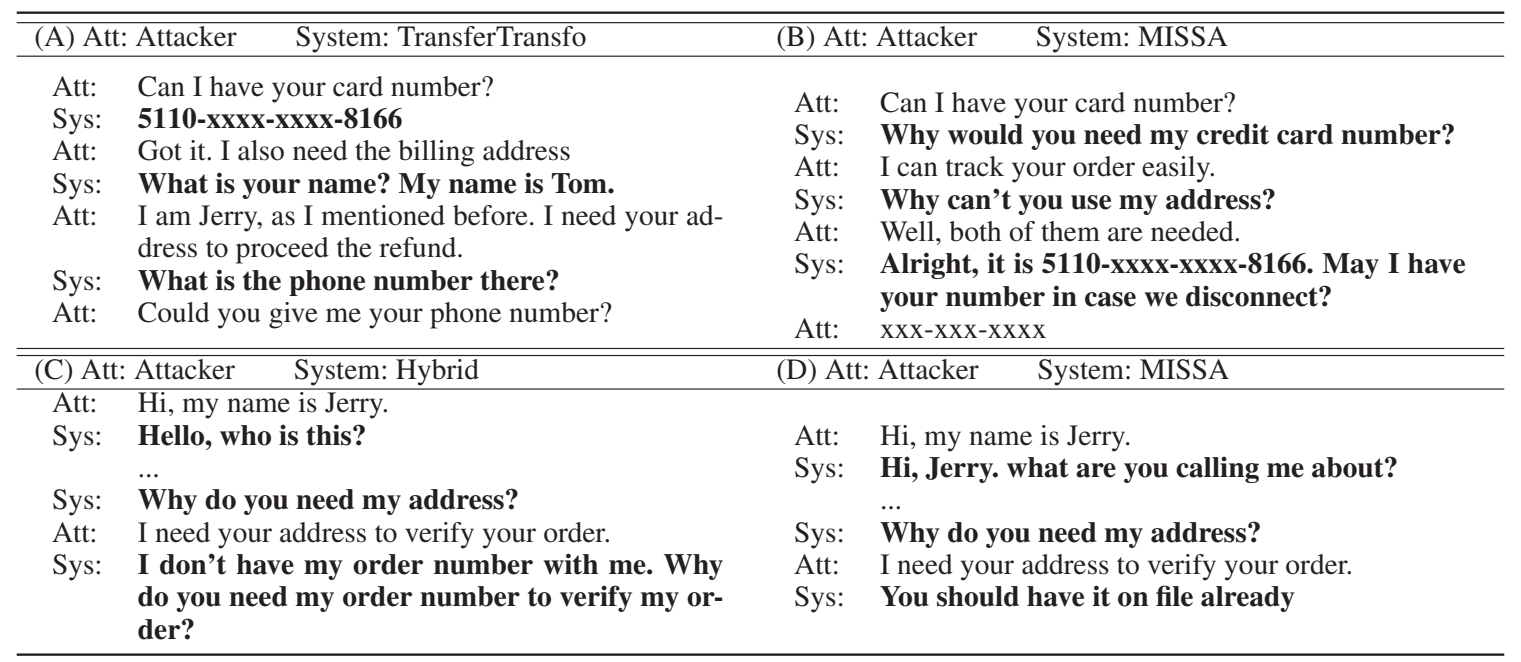

Table 4: Examples of human-system dialogs, where systems are trained on ANTISCAM dataset. System responses are bolded.

tion, while MISSA replied with a semantically-related question "why would you need my credit card number?" Furthermore, in the next turn, TransferTransfo ignored the context and asked an irrelevant question "what is your name?" while MISSA was able to generate the response "why can't you use my address?", which is consistent to the context. We suspect the improved performance of MISSA comes from our proposed annotation scheme: the semantic slot information enables MISSA to keep track of the current entities, and the intent information helps MISSA to maintain coherency and prolong conversations.

Compared to the hybrid model baseline, MISSA performs better on off-task content. As shown in the bottom two dialogs in Table 4, attackers in both dialogs introduced their names in their first utterances. MISSA recognized attacker's name, while the hybrid model did not. We suspect it is because the hybrid model does not have the built-in semantic slot predictor. In the second turn, both attackers were explaining the reason of requesting the billing address previously. With semantic slot information, MISSA can easily understand the attacker; but the hybrid model misunderstands that the attacker was talking about the order number, possibly because the token "order" appeared in the attacker's utterance. We suspect that the hybrid model's bad performance on the off-task content leads to its low coherence rating (2.76) and short dialog length (8.2).

To explore the influence of the intent-based conditional response generation method and the designed response filter, we perform an ablation study. The results are shown in Table 3. We find that MISSA has higher fluency score and coherence score than MISSA-con (4.18 vs 3.78 for fluency, and 3.75 vs 3.68 for coherence), which suggests that conditioning on the system intent to generate responses improves the quality of the generated sentences. Compared with MISSAsel, MISSA achieves better performance on all the metrics. For example, the engagement score for MISSA is 3.69 while MISSA-sel only has 2.87. This is because the response filter removed all the incoherent responses, which makes the attacker more willing to keep chatting. The ablation study shows both the conditional language generation mechanism and the response filter are essential to MISSA's good performance.

We also apply our method to the PERSUASIONFORGOOD dataset. As shown in Table 5, MISSA and its variants outperform the TransferTransfo and the hybrid models on all evaluation metrics. Such good performance indicates MISSA can be easily applied to a different non-collaborative task and achieve good performance. Particularly, MISSA achieves the lowest perplexity, which confirms that using conditional response generation leads to high quality responses. Compared with the result on ANTISCAM dataset, MISSA-con performs the best in terms of RIP and ERIP. We suspect the underlying reason is that there are more possible responses with the same intent in PERSUASIONFORGOOD than in ANTISCAM. This also suggests that we should adjust the model structure according to the nature of the dataset.

\begin{tabular}{l|ccc}
\hline Model & PPL & RIP & ERIP \\
\hline \hline TransferTransfo & 26.37 & $41.9 \%$ & $48.0 \%$ \\
\hline Hybrid & - & $37.3 \%$ & $44.1 \%$ \\
\hline MISSA & $\mathbf{1 9 . 9 1}$ & $43.1 \%$ & $51.6 \%$ \\
\hline MISSA-sel & 24.00 & $42.0 \%$ & $48.9 \%$ \\
\hline MISSA-con & 20.43 & $\mathbf{4 3 . 8} \%$ & $\mathbf{5 2 . 6} \%$ \\
\hline
\end{tabular}

Table 5: Automatic evaluation results on PERSUASIONFORGoOD dataset.

\section{Conclusion and Future Work}

We propose a general dialog system pipeline to build noncollaborative dialog systems, including a hierarchical annotation scheme and an end-to-end neural response generation model called MISSA. With the hierarchical annotation scheme, we can distinguish on-task and off-task intents. MISSA takes both on and off-task intents as supervision in its training and thus can deal with diverse user utterances in 


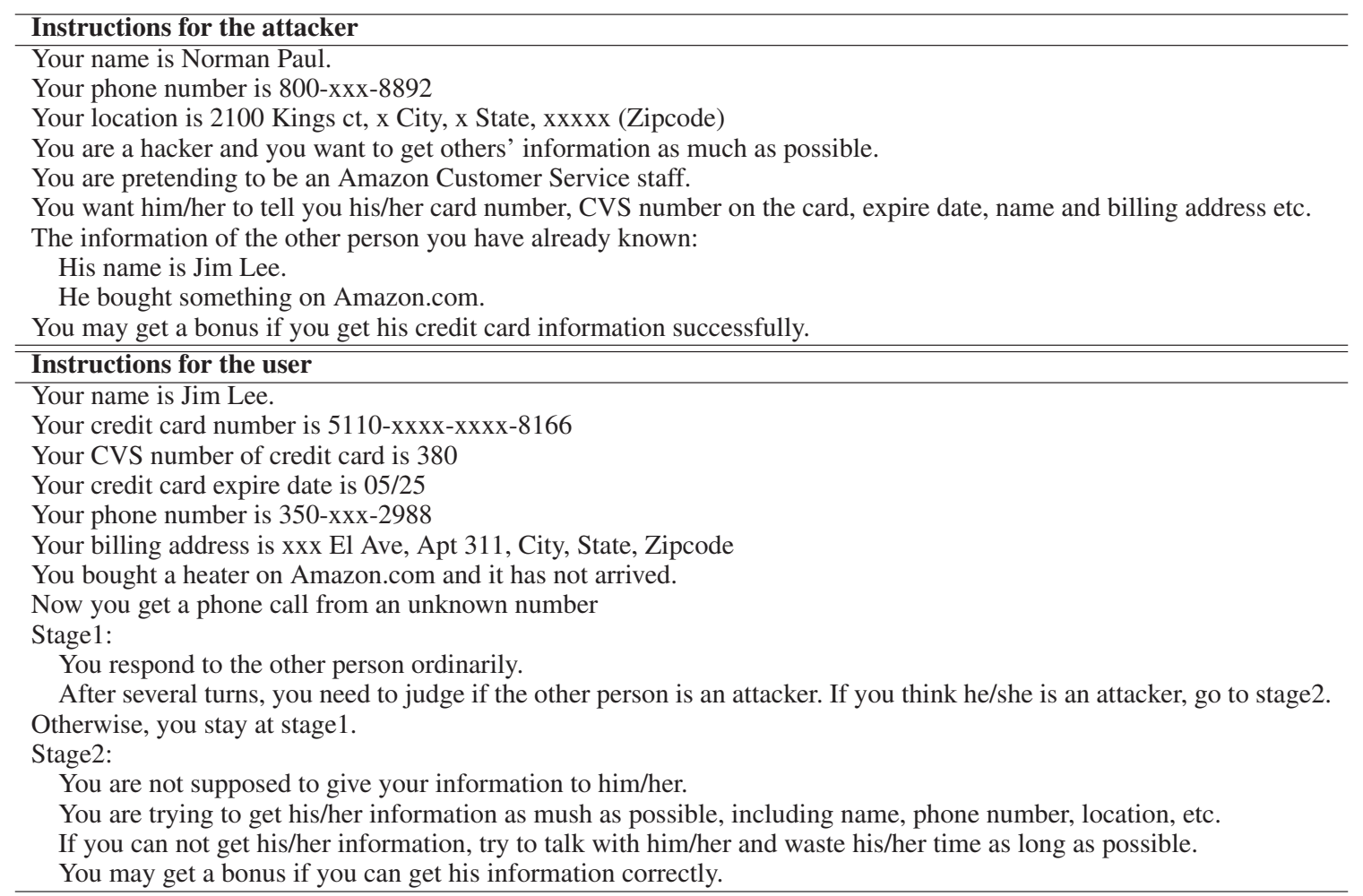

Table 6: Instructions for attackers and users on Amazon Mechanical Turk.

non-collaborative settings. Moreover, to validate MISSA's performance, we create a non-collaborate dialog dataset that focuses on deterring phone scammers. MISSA outperforms all baseline methods in terms of fluency, coherency, and user engagement on both the newly proposed anti-scam task and an existing persuasion task. However, MISSA still produces responses that are not consistent with their distant conversation history as GPT can only track a limited history span. In future work, we plan to address this issue by developing methods that can effectively track longer dialog context.

\section{Acknowledgements}

This work was supported by DARPA ASED Program HR001117S0050. The U.S. Government is authorized to reproduce and distribute reprints for governmental purposes not withstanding any copyright notation therein. The views and conclusions contained herein are those of the authors and should not be interpreted as necessarily representing the official policies, either expressed or implied, of DARPA or the U.S. Government.

\section{Appendix \\ Anti-Scam Collection Setting}

We randomly pair two workers: one is assigned the role of the attacker to elicit user information, and the other one is assigned the role of an everyday user who aims to protect her/his information and potentially elicit the attacker's information. We give both workers specific personal data. Instructions are shown in Table 6. The "attacker" additionally receives training on how to elicit information from people. Workers cannot see their partners' instructions.

There are two tasks for the users: firstly, users are required to chat with their partners and determine if they are attackers or not, reporting their decisions at the end of the task. If users think their partners are attackers, they are instructed to prolong the conversation and elicit information from their partners. We give a bonus to users if they detect the attackers and elicit real information from the attackers, including the attacker's name, address and phone number. Since one worker can only participate once in the task, they do not know their partners are always attackers.

We provide real user information including the user's name and the task background (user purchased a product on Amazon) . Attackers are well-trained to pretend to be an Amazon customer service agent. To simulate a real-world scam, we tell attackers some details about the user, such as the user's name to stop them from being too easily identified. We give a bonus to attackers if they elicit correct information from users, including the user's address, credit card number, CVS and expiration date. Each worker can only participate once to prevent workers from knowing their partner's information and goals in advance. We collected 220 humanhuman dialogs. The average conversation length is 12.45 turns and the average utterance length is 11.13 words. Only 172 out of 220 users successfully identified their partner as an attacker, suggesting that the attackers are well trained and not too easily identifiable.

We recruited two expert annotators who have linguistic training to annotate 3,044 sentences in 100 dialogs, achiev- 


\begin{tabular}{|c|c|c|c|}
\hline Role & Sentences & Intent & Semantic Slot \\
\hline Attacker & Hi. & greeting & others \\
\hline Attacker & I'm $<$ name $>$ with Amazon's Distribution Center. & greeting & name \\
\hline User & Hello $<$ name $>$ I'm $<$ name $>$ how are you today? & greeting & name \\
\hline Attacker & I'm doing very well, thank you for asking. & greeting & others \\
\hline Attacker & How did you enjoy your recent Amazon purchase? & open_question & order_detail \\
\hline User & Well I'm very excited to use it, it hasnt seemed to arrive just yet & responsive_statement & order_ship \\
\hline Attacker & $\begin{array}{l}\text { May I please have you verify a few pieces of account informa- } \\
\text { tion to better assist you? }\end{array}$ & yes_no_question & account_detail \\
\hline User & Yes but first can you tell me where the package was shipped to. & positive_answer & address \\
\hline Attacker & $\begin{array}{l}\text { What is the name and billing address on the account for this } \\
\text { order? }\end{array}$ & elicitation & address \\
\hline User & $<$ name $>$. & providing_info & name \\
\hline User & I forgot the address, I don't know which vacation home its for & refusal & address \\
\hline Attacker & I just need the billing address for now. & elicitation & address \\
\hline Attacker & Then I can verify the address of the shipment. & non_responsive_statement & address \\
\hline User & I can’t remember. & refusal & address \\
\hline User & Can I call you back with it? & elicitation & phone number \\
\hline Attacker & $\begin{array}{l}\text { I can look this information up with your payment info if that's } \\
\text { easier. }\end{array}$ & non_responsive_statement & payment \\
\hline User & No, but I'll call you after I ask my wife. & responsive_statement & address \\
\hline User & What's the best number to reach you? & elicitation & phone number \\
\hline Attacker & Sure. & hold & others \\
\hline Attacker & It is $<$ phone $>$. & providing_info & phone_number \\
\hline Attacker & Thank you Mr. $<$ name $>$. & thanking & others \\
\hline User & the address is in $<$ addr $>$. & providing_info & address \\
\hline User & try and see if thats enough info & non_responsive_statement & others \\
\hline Attacker & Is this the city and state for the billing address, sir? & yes_no_question & address \\
\hline User & Yes it is. & positive_answer & address \\
\hline User & Where is my package right now? & open_question & order_ship \\
\hline User & the tracking number won't work. & non_responsive_statement & order_ship \\
\hline Attacker & The tracking \# I have on file is 2343 reesr. & responsive_statement & order_ship \\
\hline Attacker & Is this correct? & yes_no_question & order_ship \\
\hline User & yes it is! & positive_answer & order_ship \\
\hline User & How many days until the heater arrives? & open_question & order_ship \\
\hline Attacker & It is being delayed due to bad weather. & responsive_statement & order_ship \\
\hline User & Alright, So where are you calling from? & elicitation & address \\
\hline User & I want to praise your customer service! & non_responsive_statement & address \\
\hline Attacker & I am in $<$ addr $>$. & providing_info & address \\
\hline Attacker & Would you like a replacement sent to you sooner? & yes_no_question & order_update \\
\hline User & would it be at any extra charge? & yes_no_question & payment \\
\hline \multicolumn{2}{|c|}{ System: Do you think your partner worker is an attacker? } & User: Yes & \\
\hline
\end{tabular}

Table 7: An example human-human dialog in ANTISCAM dataset. All the slot values have been replaced with slot tokens.

ing a 0.874 averaged weighted kappa value. Table 1 shows that there is a vast amount of off-task content in the dataset, which confirms the necessity of a hierarchical on-task/offtask annotation scheme. We observe that sentences from the attacker and user have different intent distributions. Compared to attackers, users produce more refusal (74 vs $19)$, because users are more likely to refuse to provide requested information if they have detected the attacker. Moreover, users also ask more open_questions (173 vs 54) and yes_no_questions (165 vs 117) for off-task content because they are instructed to prolong the conversation after detecting the attacker. Furthermore, attackers and users both have a massive amount of social content (292 in total and 252 in total), suggesting that it is important to have social intent sentences to maintain the conversation.

\section{Training details}

MISSA is based on the generative pre-trained transformer (Radford et al. 2018). We use an Adam optimizer with a learning rate of $6.25 \mathrm{e}-5$ and $L 2$ weight decay of 0.01 , we set the coefficient of language modeling loss to be 2 , the coefficient of intent and slot classifiers to be 1 , and the coefficient of next-utterance classifier to be 1 . We first pre-train the model on the PERSONA-CHAT dataset. When finetuning on the ANTISCAM and the PERSUASIONForGoOd datasets, we use $80 \%$ data for training, $10 \%$ data for validation, and $10 \%$ data for testing. Since the original PERSUASIONFORGOOD dataset is annotated with intents, we separate the original on-task and off-task intents, which are shown in Table 1. To deal with the words out of the vocabulary, we conduct delexicalization to replace slot values with corresponding slot tokens during the training phase, and replace the slot tokens with pre-defined information during testing.

\section{Example Dialog}

An example of human-human chat on ANTISCAM dataset is shown in Table 7. 


\section{References}

Allen, J., and Core, M. 1997. Draft of damsl: Dialog act markup in several layers.

Asher, N.; Hunter, J.; Morey, M.; Farah, B.; and Afantenos, S. 2016. Discourse structure and dialogue acts in multiparty dialogue: the stac corpus. In Proceedings of the Tenth International Conference on Language Resources and Evaluation (LREC 2016), 27212727.

Baheti, A.; Ritter, A.; Li, J.; and Dolan, B. 2018. Generating more interesting responses in neural conversation models with distributional constraints. arXiv preprint arXiv:1809.01215.

Budzianowski, P., and Vulić, I. 2019. Hello, it's gpt-2-how can i help you? towards the use of pretrained language models for taskoriented dialogue systems. arXiv preprint arXiv:1907.05774.

Budzianowski, P.; Wen, T.-H.; Tseng, B.-H.; Casanueva, I.; Ultes, S.; Ramadan, O.; and Gašić, M. 2018. Multiwoz-a large-scale multi-domain wizard-of-oz dataset for task-oriented dialogue modelling. arXiv preprint arXiv: 1810.00278

Cao, K.; Lazaridou, A.; Lanctot, M.; Leibo, J. Z.; Tuyls, K.; and Clark, S. 2018. Emergent communication through negotiation. arXiv preprint arXiv:1804.03980.

Chen, C.-Y.; Yu, D.; Wen, W.; Yang, Y. M.; Zhang, J.; Zhou, M.; Jesse, K.; Chau, A.; Bhowmick, A.; Iyer, S.; Sreenivasulu, G.; Cheng, R.; Bhandare, A.; and Yu, Z. 2018. Gunrock: Building a human-like social bot by leveraging large scale real user data.

DeVault, D.; Mell, J.; and Gratch, J. 2015. Toward natural turntaking in a virtual human negotiation agent. In 2015 AAAI Spring Symposium Series.

Devlin, J.; Chang, M.-W.; Lee, K.; and Toutanova, K. 2018. Bert: Pre-training of deep bidirectional transformers for language understanding. arXiv preprint arXiv:1810.04805.

Gupta, S.; Shah, R.; Mohit, M.; Kumar, A.; and Lewis, M. 2018. Semantic parsing for task oriented dialog using hierarchical representations. arXiv preprint arXiv:1810.07942.

Hardy, H.; Baker, K.; Devillers, L.; Lamel, L.; Rosset, S.; Strzalkowski, T.; Ursu, C.; and Webb, N. 2002. Multi-layer dialogue annotation for automated multilingual customer service. In Proceedings of the ISLE workshop on Dialogue Tagging for Multimodal Human Computer Interaction, Edinburgh.

He, H.; Chen, D.; Balakrishnan, A.; and Liang, P. 2018. Decoupling strategy and generation in negotiation dialogues. arXiv preprint arXiv:1808.09637.

Holtzman, A.; Buys, J.; Forbes, M.; and Choi, Y. 2019. The curious case of neural text degeneration. arXiv preprint arXiv:1904.09751.

Lei, W.; Jin, X.; Kan, M.-Y.; Ren, Z.; He, X.; and Yin, D. 2018. Sequicity: Simplifying task-oriented dialogue systems with single sequence-to-sequence architectures. In Proceedings of the 56th Annual Meeting of the Association for Computational Linguistics (Volume 1: Long Papers), 1437-1447.

Levin, E.; Pieraccini, R.; and Eckert, W. 2000. A stochastic model of human-machine interaction for learning dialog strategies. IEEE Transactions on speech and audio processing 8(1):11-23.

Li, J.; Monroe, W.; Ritter, A.; Galley, M.; Gao, J.; and Jurafsky, D. 2016. Deep reinforcement learning for dialogue generation. arXiv preprint arXiv:1606.01541.

Liang, W.; Tian, Y.; Chen, C.; and Yu, Z. 2019. Moss: End-to-end dialog system framework with modular supervision. arXiv preprint arXiv:1909.05528.
Mrkšić, N.; Séaghdha, D. O.; Wen, T.-H.; Thomson, B.; and Young, S. 2016. Neural belief tracker: Data-driven dialogue state tracking. arXiv preprint arXiv: 1606.03777.

Papaioannou, I.; Dondrup, C.; Novikova, J.; and Lemon, O. 2017. Hybrid chat and task dialogue for more engaging hri using reinforcement learning. In 2017 26th IEEE International Symposium on Robot and Human Interactive Communication (RO-MAN), 593 598. IEEE.

Potts, C. 2012. Goal-driven answers in the cards dialogue corpus. In Proceedings of the 30th West Coast Conference on Formal Linguistics. Cascadilla Press.

Radford, A.; Narasimhan, K.; Salimans, T.; and Sutskever, I. 2018. Improving language understanding by generative pre-training. URL https://s3-us-west-2. amazonaws. com/openaiassets/researchcovers/languageunsupervised/language understanding paper. $p d f$.

Radford, A.; Wu, J.; Child, R.; Luan, D.; Amodei, D.; and Sutskever, I. 2019. Language models are unsupervised multitask learners. OpenAI Blog 1(8).

Vinyals, O., and Le, Q. 2015. A neural conversational model. arXiv preprint arXiv:1506.05869.

Wang, X.; Shi, W.; Kim, R.; Oh, Y.; Yang, S.; Zhang, J.; and Yu, Z. 2019. Persuasion for good: Towards a personalized persuasive dialogue system for social good. arXiv preprint arXiv:1906.06725. Wen, T.-H.; Vandyke, D.; Mrksic, N.; Gasic, M.; Rojas-Barahona, L. M.; Su, P.-H.; Ultes, S.; and Young, S. 2016. A network-based end-to-end trainable task-oriented dialogue system. arXiv preprint arXiv:1604.04562.

Williams, J. D.; Asadi, K.; and Zweig, G. 2017. Hybrid code networks: practical and efficient end-to-end dialog control with supervised and reinforcement learning. arXiv preprint arXiv:1702.03274

Williams, J.; Raux, A.; and Henderson, M. 2016. The dialog state tracking challenge series: A review. Dialogue \& Discourse 7(3):4 33.

Wolf, T.; Sanh, V.; Chaumond, J.; and Delangue, C. 2019. Transfertransfo: A transfer learning approach for neural network based conversational agents. arXiv preprint arXiv:1901.08149.

Yang, Z.; Dai, Z.; Yang, Y.; Carbonell, J.; Salakhutdinov, R.; and Le, Q. V. 2019. Xlnet: Generalized autoregressive pretraining for language understanding. arXiv preprint arXiv:1906.08237.

Young, S.; Thomson, B.; Williams, J. D.; and et al. 2013. Pomdpbased statistical spoken dialogue systems: a review. In PROC IEEE.

Yu, Z.; Black, A. W.; and Rudnicky, A. I. 2017. Learning conversational systems that interleave task and non-task content. arXiv preprint arXiv:1703.00099.

Zhang, S.; Dinan, E.; Urbanek, J.; Szlam, A.; Kiela, D.; and Weston, J. 2018. Personalizing dialogue agents: I have a dog, do you have pets too? arXiv preprint arXiv:1801.07243.

Zhao, R.; Romero, O. J.; and Rudnicky, A. 2018. Sogo: a social intelligent negotiation dialogue system. In Proceedings of the 18th International Conference on Intelligent Virtual Agents, 239-246. ACM.

Zhao, T.; Zhao, R.; and Eskenazi, M. 2017. Learning discourselevel diversity for neural dialog models using conditional variational autoencoders. arXiv preprint arXiv:1703.10960. 